\title{
Four-dimensional de Sitter space is a Glauber-Sudarshan state in string theory
}

\author{
Suddhasattwa Brahma, ${ }^{a}$ Keshav Dasgupta ${ }^{a}$ and Radu Tatar ${ }^{b}$ \\ ${ }^{a}$ Department of Physics, McGill University, \\ Montréal, QC H3A 2T8, Canada \\ ${ }^{b}$ Department of Mathematical Sciences, University of Liverpool, \\ Liverpool, L69 7ZL, U.K. \\ E-mail: suddhasattwa.brahma@gmail.com, keshav@hep.physics.mcgill.ca, \\ Radu.Tatar@Liverpool.ac.uk
}

Abstract: We show that four-dimensional de Sitter space is a Glauber-Sudarshan state, i.e. a coherent state, over a supersymmetric solitonic background in full string theory. We argue that such a state is only realized in the presence of temporally varying degrees of freedom and after including quantum corrections, with supersymmetry being broken spontaneously. On the other hand, fluctuations over the resulting de Sitter space is governed by the Agarwal-Tara state, which is a graviton (and flux)-added coherent state. Once de Sitter space is realized as a coherent state, and not as a vacuum, its ability to remain out of the swampland as well as issues regarding its (meta)stability, vacuum energy, and finite entropy appear to have clear resolutions.

KEywords: M-Theory, Flux compactifications

ArXiv EPrint: 2007.00786 


\section{Contents}

1 Introduction \& setup 1

2 The Glauber-Sudarshan state $\quad 3$

3 Fluctuations \& the Agarwal-Tara state $\quad 6$

$\begin{array}{lll}4 & \text { Stability } & 7\end{array}$

$\begin{array}{llr}5 & \text { Conclusion } & 9\end{array}$

\section{Introduction \& setup}

The search for four-dimensional de Sitter (dS) has led to claims varying from having numerous solutions [1-4] to having none [5-8]. The standard picture of a landscape of vacua [9], along with an anthropic principle to explain our own universe [10], runs into problems not because of any no-go theorem [11-14], or technical hindrances [15, 16], but due to obstructions against dS spacetimes ingrained in some fundamental aspects of quantum gravity [17-22]. These include trans-Planckian issues [23] challenging the very notion of a well-defined, unitary Wilsonian effective action for accelerating backgrounds [17-19], or that of instabilities associated with the vacuum state for dS leading to a quantum swampland [24]. It was recently shown that the hurdles related to an effective field theory description of dS, as summarized in [30], can, in principle, be overcome once time-dependent degrees are switched on [25-29]. In this work, we show that other fundamental obstructions are also avoided if four-dimensional dS space itself is regarded as a coherent state over a Minkowski spacetime. Although similar setups have been considered before [31-35], what is new here is an explicit construction of such a configuration realized in full string theory. ${ }^{1}$ Remarkably, the resulting construction of dS space hints at natural resolutions to problems related to its stability, vacuum energy and entropy.

We shall focus on $\mathrm{dS}$ in the flat slicing, with a metric of the form:

$$
\mathrm{d} s^{2}=\frac{1}{\Lambda|t|^{2}}\left(-\mathrm{d} t^{2}+\mathrm{d} x_{1}^{2}+\mathrm{d} x_{2}^{2}+\mathrm{d} x_{3}^{2}\right)
$$

where $\Lambda$ is the cosmological constant and the temporal coordinate $t$ has a range $-\infty \leq t \leq 0$, with the late time regime given by $t \rightarrow 0$. We choose this specific realization of $\mathrm{dS}$ (1.1) only for computational efficiency and other realizations, specifically of Kasner-type, have

\footnotetext{
${ }^{1} \mathrm{~A}$ more detailed version, with proofs and computations, appears in [36].
} 
been considered earlier [25]. The question is how to realize a metric like (1.1) in, say, type IIB string theory? A natural first conjecture is the following metric configuration:

$$
\mathrm{d} s^{2}=\frac{1}{\Lambda \mathrm{H}^{2}(y)|t|^{2}}\left(-\mathrm{d} t^{2}+\mathrm{d} x_{i}^{2}\right)+\mathrm{H}^{2}(y) g_{\mathrm{MN}}(y) \mathrm{d} y^{\mathrm{M}} \mathrm{d} y^{\mathrm{N}},
$$

where $\mathrm{H}(y)$ is the warp-factor that only depends on the coordinate of the internal sixdimensional manifold whose unwarped metric is given by $g_{\mathrm{MN}}(y)$. Crucially, note that the internal space is time-independent. To see what kind of fluxes are required to support a configuration like (1.2) in IIB string theory, we uplift the configuration to M-theory. This uplift is only for computational advantage, and has no deeper implications as the degrees of freedom remain unchanged. The uplifted metric becomes:

$$
\mathrm{d} s^{2}=g_{s}^{-8 / 3} \eta_{\mu \nu} \mathrm{d} x^{\mu} \mathrm{d} x^{\nu}+g_{s}^{-2 / 3} \mathrm{H}^{2} g_{\mathrm{MN}} \mathrm{d} y^{\mathrm{M}} \mathrm{d} y^{\mathrm{N}}+g_{s}^{4 / 3}|\mathrm{~d} z|^{2},
$$

where $g_{s} \equiv \sqrt{\Lambda}|t| \mathrm{H}(y)$ is the IIA string coupling which is now a function of both $y^{\mathrm{M}}$ and $t$, implying that in M-theory the internal manifold becomes time-dependent and $g_{s} \rightarrow 0$ denotes late time. Additionally, since we require $\left(g_{s} / \mathrm{H}\right)<1$, our analysis would only make sense in the interval:

$$
-\frac{1}{\sqrt{\Lambda}}<t<0
$$

Beyond this limit, we lose all quantitative control on the dynamics. Consequently, this means that not only is our solution trustworthy only below the quantum break-time of dS [22] and the TCC limit [17], but it also provides a simple resolution of the coincidence problem of dark energy by limiting the age of our universe by the current Hubble time [37], and avoids problems of Boltzmann brains.

The dynamics is controlled by metric and fluxes because, to support such a manifold, we need G-fluxes on an eleven-dimensional space whose topology is:

$$
\mathcal{M}_{11}=\mathbb{R}^{2,1} \times \mathcal{M}_{8} \equiv \mathbb{R}^{2,1} \times \mathcal{M}_{4} \times \mathcal{M}_{2} \times \frac{\mathbb{T}^{2}}{\mathcal{G}}
$$

where $(\mu, \nu)$ parametrize $\mathbb{R}^{2,1},(\mathrm{M}, \mathrm{N})$ parametrize the six-dimensional base with unwarped metric $g_{\mathrm{MN}}(y)$ and $z^{a}$ parametrizes the toroidal fibre modded out by the isometry group $\mathcal{G}$. Let $\mathbf{G}_{\mathrm{MNPQ}}$ and $\mathbf{G}_{\mathrm{MN} a b}$, where $(\mathrm{M}, \mathrm{N}) \in \mathcal{M}_{6}$ and $(a, b) \in \frac{\mathbb{T}^{2}}{\mathcal{G}}$, denote the 4-fluxes switched on in the internal space. Fluxes on a compact space cannot be arbitrary: they have to satisfy Gauss' law (or alternatively, cancel anomalies), solve the EOMs, and be quantized. The quantization condition is [38]:

$$
\left[\frac{\mathbf{G}_{4}}{2 \pi}\right]-\frac{p_{1}(y)}{4} \in \mathbb{H}^{4}(y, \mathbb{Z}),
$$

where $\mathbf{G}_{4}$ is a four-form G-flux component, $p_{1}(y)$ is the first Pontryagin class and $\mathbb{H}^{4}(y, \mathbb{Z})$ is the fourth cohomology class. In a time-dependent internal space like (1.3), this quantization condition cannot be satisfied with time-independent G-flux components. Additionally, EOMs also require fluxes to become time- dependent raising, in turn, serious questions about how could these fluxes be quantized, and how could they satisfy Gauss' law. On the dual 
IIB side, this means that the three and the five-form fluxes should become time-dependent, leading to similar questions. The axio-dilaton however remains time-independent because we are in the constant-coupling scenario of F-theory [25, 41]. In the absence of quantum corrections and time-dependent fluxes, as shown in [25], all the questions raised above cannot be answered and the system is inconsistent, stemming from the loss of $g_{s}$ and $\mathrm{M}_{p}$ hierarchies, leading to a breakdown of effective field theory as another manifestation of the swampland conjectures [30]. More importantly, a metric like (1.3) is still a bit too constrained to be a solution, and what really works is a metric of the form:

$$
\begin{aligned}
\mathrm{d} s^{2}= & g_{s}^{-8 / 3} \eta_{\mu \nu} \mathrm{d} x^{\mu} \mathrm{d} x^{\nu}+g_{s}^{-2 / 3} \mathrm{H}^{2}\left(\mathrm{~F}_{1}(t) g_{\alpha \beta} \mathrm{d} y^{\alpha} \mathrm{d} y^{\beta}\right. \\
& \left.+\mathrm{F}_{2}(t) g_{m n} \mathrm{~d} y^{m} \mathrm{~d} y^{n}\right)+g_{s}^{4 / 3}|\mathrm{~d} z|^{2}
\end{aligned}
$$

where $(\alpha, \beta) \in \mathcal{M}_{2}$ and $(m, n) \in \mathcal{M}_{4}$ of (1.5). The additional time-dependences governs how the six-dimensional manifold $\mathcal{M}_{6}=\mathcal{M}_{4} \times \mathcal{M}_{2}$ changes with respect to time. On the IIB side this converts (1.2) to the following:

$$
\begin{aligned}
\mathrm{d} s^{2}= & \frac{1}{\Lambda \mathrm{H}^{2}(y)|t|^{2}}\left(-\mathrm{d} t^{2}+\mathrm{d} x_{1}^{2}+\mathrm{d} x_{2}^{2}+\mathrm{d} x_{3}^{2}\right) \\
& +\mathrm{H}^{2}(y)\left(\mathrm{F}_{1}(t) g_{\alpha \beta}(y) \mathrm{d} y^{\alpha} \mathrm{d} y^{\beta}+\mathrm{F}_{2}(t) g_{m n}(y) \mathrm{d} y^{m} \mathrm{~d} y^{n}\right),
\end{aligned}
$$

making the internal space time-dependent. To preserve four-dimensional Newton's constant, we additionally require $\mathrm{F}_{1} \mathrm{~F}_{2}^{2}=1$ and both $\mathrm{F}_{i}(t) \rightarrow 1$ as $g_{s} \rightarrow 0$. Imposing all these conditions, the system becomes tightly constrained but does have a solution, answering all the questions that we raised above, as shown in [25]. However, even after we get an effective potential which supports dS space in string theory, there still remains questions whether the radiative corrections, calculated for some vacuum state, leads to instabilities [24] or if the obstructions regarding trans-Planckian issues [17] lingers on. To answer these, we attempt to see if a metric like (1.8) or (1.7) can be realized as a state instead of a vacuum.

\section{The Glauber-Sudarshan state}

The state that we have in mind is the Glauber-Sudarshan state [39, 40], commonly called a coherent state, because such a state is closest to a classical configuration which can be realized in a quantum theory. Our aim would be to realize (1.7) as a Glauber-Sudarshan state over a supersymmetric solitonic vacuum. (Then, by dualization, (1.8) can also be realized as such a state.) The supersymmetric vacuum would be a warped Minkowski space. The supersymmetric solitonic background that we have in mind is of the form:

$$
\mathrm{d} s^{2}=\frac{1}{h^{2 / 3}(y)}\left(-\mathrm{d} t^{2}+\mathrm{d} x_{i}^{2}\right)+h^{1 / 3}(y) g_{\mathrm{MN}}^{(0)} \mathrm{d} y^{\mathrm{M}} \mathrm{d} y^{\mathrm{N}},
$$

where $h(y)$ is the warp-factor, and can be supported by self-dual G-fluxes of the form $\mathbf{G}_{\mathrm{MNPQ}}^{(0)}(y)$, as well as $\mathbf{G}_{0 i j \mathrm{M}}^{(0)}$, where $(\mathrm{M}, \mathrm{N}) \in \mathcal{M}_{8}$. Such a background has been studied in detail in [43]. If we study fluctuations over this background, they are classified by modes which may be easily determined. These modes will typically have non-trivial spatial 
behavior, whose dynamics will be governed by a Schrödinger equation over a non-trivial potential, but their temporal behavior would be simple $\left(\sim e^{i \omega_{\mathbf{k}} t}\right)$. This will help us to avoid any trans-Planckian issues, but new subtleties lie in the construction of the GlauberSudarshan state itself. In the original work of $[39,40]$, the state was created by shifting the free vacuum (or the harmonic vacuum) by a displacement operator. One of the main issue in our case is that there is no free vacuum in a highly interacting theory like M-theory! Secondly, M-theory has metric as well as G-flux components, so a Glauber-Sudarshan state would be more complicated to account for fluctuations of all these ingredients. Thirdly, even if we manage to construct such a state, how do we know that such a state survives the set of quantum corrections coming from perturbative, non-perturbative, non-local and topological interactions?

Let us start by answering the first question, related to the construction of the GlauberSudarshan state. Since there is no free vacuum once interactions are switched on, we only have the interacting vacuum $|\Omega\rangle$ to build our state from. We shall shift the interacting vacuum by a displacement operator and ask if this creates a state resembling the GlauberSudarshan state, namely:

$$
|\sigma\rangle \equiv \mathbb{D}(\sigma)|\Omega\rangle
$$

at a specific time $t=t_{0} . \mathbb{D}(\sigma)$ is the displacement operator and $\sigma \equiv\left(\left\{\alpha_{g}\right\},\left\{\beta_{\mathrm{C}}\right\}\right)$ where $\left\{\alpha_{g}\right\}$ and $\left\{\beta_{\mathrm{C}}\right\}$ are the two sets of parameters associated with all the metric and the Cfield components, respectively. The definition of a displacement operator in an interacting theory is, however, not clear. In the free theory, a displacement operator is constructed from annihilation $a_{\mathbf{k}}$ and creation $a_{\mathbf{k}}^{\dagger}$ operators for a given spatial momentum $\mathbf{k}$, so in an interacting theory we expect analogous operators $a_{\mathrm{eff}}(\mathbf{k})$ and $a_{\mathrm{eff}}^{\dagger}(\mathbf{k})$ to replace them, with $a_{\text {eff }}(\mathbf{k})$ annihilating the interacting vacuum. Unfortunately this information is not enough to fix the form of $a_{\mathrm{eff}}(\mathbf{k})$, the latter being is a complicated function that mixes the freefield annihilation and creation operators for different spatial momenta [36]. Due to this, $\mathbb{D}(\sigma)$ is not a unitary operator anymore. However, there does exist one possible choice for $\mathbb{D}(\sigma)$ that not only fixes the form for $a_{\text {eff }}(\mathbf{k})$, but also reproduces the background (1.7) as expectation values of the metric operators in the state (2.2). This choice works for any time $t$ and is given by:

$$
\mathbb{D}(\sigma, t)=\mathbb{D}_{0}(\sigma) \exp \left(i \int_{-T}^{t} d^{11} x \mathbf{H}_{\text {int }}\right),
$$

where $T \rightarrow \infty$ in a slightly imaginary direction; and $\mathbf{H}_{\mathrm{int}} \equiv \mathbf{H}_{\mathrm{int}}\left(g_{\mathrm{MN}}, \mathrm{C}_{\mathrm{PQR}}\right)$ is the full interacting part of the M-theory Hamiltonian. $\mathbb{D}_{0}(\sigma)$ is the displacement operator for the harmonic vacuum, meaning that it displaces the harmonic vacuum by $\sigma \equiv \sigma(t)$ to create the required Glauber-Sudarshan state with one minor difference: it is the non-unitary part of the usual free vacuum displacement operator. In writing (2.3) we have ignored a multiplicative constant piece that is proportional to the overlap between the interacting and the harmonic vacuum, i.e. $\langle\Omega \mid 0\rangle$. One can also work out the wavefunction in the configuration space for the state (2.2) satisfying (2.3). For example, say, for the spacetime 
mode for the graviton, the wave-function of the state (2.2) may be expressed explicitly as:

$$
\Psi_{\Omega}^{(\sigma)}\left(g_{\mu \nu}, t\right)=\exp \left[\int_{-\infty}^{+\infty} d^{10} \mathbf{k} \log \left\langle\widetilde{g}_{\mu \nu}(\mathbf{k}) \mid \Psi_{\mathbf{k}}^{(\sigma)}(t)\right\rangle\right]
$$

where $\left|\Psi_{\mathbf{k}}^{(\sigma)}(t)\right\rangle$ is the Glauber-Sudarshan state in the Heisenberg representation for a given spatial mode $\mathbf{k}$ and $\widetilde{g}_{\mu \nu}(\mathbf{k})$ is the Fourier component of the graviton.

Indeed, this is all we need for the present purpose, as all of the background quantities simply appear by taking expectation values over the state (2.2) with the wavefunction (2.4). For example, let us concentrate on the space-time metric again. The expectation value of the metric operator may be expressed as:

$$
\left\langle\mathbf{g}_{\mu \nu}\right\rangle_{\sigma}=\frac{\int\left[\mathcal{D} g_{\mu \nu}\right] e^{i \mathbf{S}_{\mathbb{D}^{\dagger}}(\sigma) g_{\mu \nu} \mathbb{D}(\sigma)}}{\int\left[\mathcal{D} g_{\mu \nu}\right] e^{i \mathbf{S}^{\dagger}} \mathbb{D}^{\dagger}(\sigma) \mathbb{D}(\sigma)}=\frac{\eta_{\mu \nu}}{\left(\Lambda|t|^{2} \mathrm{H}^{2}(y)\right)^{4 / 3}}
$$

where $\mathbf{S} \equiv \mathbf{S}\left(g_{\mathrm{MN}}, \mathrm{C}_{\mathrm{PQR}}\right)$ is the total M-theory action. Since $\mathbb{D}(\sigma) \equiv \mathbb{D}(\sigma(t), t)$ is nonunitary, not only does it necessitate a division by another path integral as shown in the middle equality, but also it keeps the numerator from vanishing. In fact, $\mathbb{D}(\sigma)$ does what it is expected to do: it shifts the vacuum in such a way that the one-point functions do not vanish and ensures that they have the necessary expectation values. In addition, the choice (2.3) guarantees that there are no $\mathcal{O}\left(\frac{g_{s}^{a}}{\mathrm{M}_{p}^{b}}\right)$ corrections to (2.5) [36]. The above computation relies on two essential objects: (i) the space-time wave-functions (not the configuration space wave-functions!) which come from solving a class of Schrödinger equations with non-trivial potentials, and (ii) the Glauber-Sudarshan wave-function (2.4). Similarly, the expectation value of the G-flux components over the state (2.2) becomes:

$$
\left\langle\mathbf{G}_{\mathrm{MNPQ}}\right\rangle_{\sigma}=\sum_{p} \mathcal{G}_{\mathrm{MNPQ}}^{(p)}(y)\left(\frac{g_{s}}{\mathrm{H}}\right)^{2 p / 3},
$$

where $(\mathrm{M}, \mathrm{N}) \in \mathcal{M}_{8}$ with $p \in \frac{\mathbb{Z}}{2}$ and $p \geq \frac{3}{2}$. The latter condition stems from various criteria, including flux EOMs, Bianchi identities and subtleties with localized fluxes, as elaborated in [25]. We want to emphasize that the bound on $p$ tells us that there are no time-independent fluxes allowed in this set-up.

Note that supersymmetry is broken spontaneously because the G-flux on the internal space is no longer self-dual, i.e.

$$
\left|\left\langle\mathbf{G}_{4}\right\rangle_{\sigma}-\left\langle *_{8} \mathbf{G}_{4}\right\rangle_{\sigma}\right|>0
$$

where $\mathbf{G}_{4}$ is the four-form operator and $*_{8}$ is the Hodge dual with respect to the un-warped metric of the solitonic background (2.1). Note that the fluxes $\mathbf{G}_{4}^{(0)}$, supporting (2.1), are self dual and preserve supersymmetry for the solitonic vacuum (thereby canceling the zero-point energies) and, subsequently, supersymmetry is broken spontaneously by the Glauber-Sudarshan state. The cosmological constant is much lower than the energy scale of supersymmetry breaking due to suppression by a factor of the unwarped volume [36]. 


\section{Fluctuations \& the Agarwal-Tara state}

Next, to study instabilities associated with QFT in dS, we wish to explore fluctuations over dS space in such a setting. Since dS itself is a state over the solitonic vacuum, the fluctuations should also come from a related state appearing as some deformation of the Glauber-Sudarshan state. It turns out that the required deformation is another well-known state, called the Agarwal-Tara state [45], or alternatively, as the graviton added coherent state. For us, this needs to be generalized in the same vein as the Glauber-Sudarshan state so that the Agarwal-Tara state will have to be both graviton and flux-added coherent state, which is given by:

$$
\left|\Psi_{\mathbf{k}}^{\left(c_{1} c_{2}\right)}(t)\right\rangle=\left[c_{1}+c_{2} \mathcal{G}\left(a_{\mathbf{k}}+a_{\mathbf{k}}^{\dagger} ; t\right)\right]\left|\Psi_{\mathbf{k}}^{(\sigma)}(t)\right\rangle,
$$

where we have used (2.3), and denote $a_{\mathrm{eff}}(\mathbf{k})$ and $a_{\mathrm{eff}}^{\dagger}(\mathbf{k})$ by $a_{\mathbf{k}}$ and $a_{\mathbf{k}}^{\dagger}$ to simplify the notation, and $c_{i}$ are constants with $\left|c_{2}\right| \ll\left|c_{1}\right|$. We have further restricted to the graviton sector to avoid over-burdening the formula with complications from the flux sector. $\mathcal{G}(w, t)$ could be thought of as a polynomial function of $w$ with time-dependent coefficient (details appear in [36]). Subtleties aside, what is significant for us is that the expectation of the graviton operator in such a state gives us:

$$
\begin{aligned}
\left\langle\mathbf{g}_{\mu \nu}\right\rangle_{\Psi^{\left(c_{1} c_{2}\right)}}= & \frac{\eta_{\mu \nu}}{\left(\Lambda|t|^{2} \mathrm{H}^{2}(y)\right)^{4 / 3}} \\
& +c_{2} \int d^{11} k h\left(\mathbf{k}, k_{0}\right) \alpha_{\mu \nu}\left(\mathbf{k}, k_{0}\right) \psi_{\mathbf{k}}(\mathbf{x}, y, z) e^{i k_{0} t},
\end{aligned}
$$

where $\alpha_{\mu \nu}$ is precisely the parameter from the set $\left\{\alpha_{g}\right\}$ of $\sigma \equiv\left(\left\{\alpha_{g}\right\},\left\{\beta_{\mathrm{C}}\right\}\right)$ that defines the Glauber-Sudarshan state (2.2). $\psi_{\mathbf{k}}(\mathbf{x}, y, z)$ is the eleven-dimensional spatial wave-function that appears from the Schrödinger equation over the solitonic background alluded to earlier. We have also taken $c_{1}=1$, so only $c_{2}$ appears in $(3.2) . h\left(\mathbf{k}, k_{0}\right)$ is the Fourier component that comes from $\mathcal{G}(w, t)$ function in (3.1). The crucial take-home point from (3.2) is that the temporally varying frequencies $\omega_{\mathbf{k}}(t)$ that we get from fluctuations over a dS vacuum are nothing but artifacts of the Fourier transforms over the Glauber-Sudarshan state. In other words:

$$
\exp \left(-i \omega_{\mathbf{k}}(t) t\right) \equiv \int d k_{0} h\left(\mathbf{k}, k_{0}\right) e^{-i k_{0} t},
$$

where $\omega_{\mathbf{k}}(t)$ is in general a complex function. Such a conclusion seems to point out that there are no trans-Planckian censorship required for our construction [17] because all modes originate secretly from fluctuations over our solitonic background (2.1). (We also satisfy the conditions of having a underlying Lorentz-invariant spacetime, with a local vacuum, required to avoid the original trans-Planckian problem [23].) As a result, Wilsonian analysis can be performed for these fluctuations because all modes in our theory can be expressed as linear combinations of the ones over the solitonic background with time-independent frequencies. Since our dS space, itself, is a state which results from these modes, it is no surprise that we also get fluctuations on top of dS as another consequence of them. However, we still need to demonstrate the stability of our construction, which we shall address next. 


\section{Stability}

How do we know that the quantum corrections do not take us away from the background (1.7) and G-flux (2.6) configurations? In fact, any small changes to the $\mathrm{F}_{i}(t)$ factors in (1.7) will switch on some time-dependence of the Newton's constant. In order to avoid this, we have to establish the stability of the solution. This also gets tied up with the equations of motion in the presence of all possible quantum corrections. It turns out that the background equations may be presented most succinctly as Schwinger-Dyson equations (SDEs). The SDEs [46-48] are expressed as expectation values over the Glauber-Sudarshan state and therefore suits our construction very well since we have considered the metric, the G-fluxes as well as the fluctuations as expectation values. The SDEs for our case may be divided into two set. One set is easy to write down and is given by:

$$
\frac{\delta \mathbf{S}^{(\sigma)}}{\delta\left\langle g^{\mathrm{MN}}\right\rangle_{\sigma}}=\frac{\delta \mathbf{S}^{(\sigma)}}{\delta\left\langle\mathrm{C}^{\mathrm{MNP}}\right\rangle_{\sigma}}=0,
$$

where $\mathbf{S}^{(\sigma)} \equiv \mathbf{S}^{(\sigma)}\left(\left\langle g_{\mathrm{MN}}\right\rangle_{\sigma},\left\langle\mathrm{C}_{\mathrm{PQR}}\right\rangle_{\sigma}\right)$ differs from $\mathbf{S}=\mathbf{S}\left(g_{\mathrm{MN}}, \mathrm{C}_{\mathrm{PQR}}\right)$ in

$$
\left\langle\mathbf{g}_{\mu \nu}\right\rangle_{\sigma}=\frac{\int\left[\mathcal{D} g_{\mu \nu}\right] e^{i \mathbf{S}_{\mathbb{D}^{\dagger}}(\sigma) g_{\mu \nu} \mathbb{D}(\sigma)}}{\int\left[\mathcal{D} g_{\mu \nu}\right] e^{i \mathbf{S}^{\dagger}} \mathbb{D}^{\dagger}(\sigma) \mathbb{D}(\sigma)}=\frac{\eta_{\mu \nu}}{\left(\Lambda|t|^{2} \mathrm{H}^{2}(y)\right)^{4 / 3}},
$$

by the appearance of the expectation values in the integrands themselves. The second set of equations are however more involved and they include both the Faddeev-Popov ghosts and the displacement operator [36]. For us (4.1) will suffice, as the form of these equations precisely imply the equations of motion already studied in [25] and, therefore, pursues the path laid down there, as follows. Once we express the equations order by order in $\left(\frac{g_{s}}{\mathrm{H}}\right)$, the zeroth order equations precisely determine the background (1.7) with the G-fluxes as in (2.6). Going to the higher orders in $\left(\frac{g_{s}}{\mathrm{H}}\right)$ then switches on three things: (a) higher orders, i.e $p>\frac{3}{2}$ in (2.6) for the G-fluxes, (b) higher order terms for the $\mathrm{F}_{i}(t)$ factors in (1.7), and (c) higher order quantum terms discussed in [25]. Together, they balance each other in such a way that the zeroth order metric and G-fluxes, from (1.7) and (2.6) respectively, do not receive any corrections [36].

We can also see how moduli can be stabilized in such time-dependent background. The time-independent fluxes and the quantum corrections that are required to support the solitonic background (2.1) also stabilize the Kähler and the complex structure moduli of the internal manifold without breaking any supersymmetry. The time-dependent background can then be generated by taking the expectation values of the metric and the G-flux operators as in (4.2) and (2.6). This means the stabilized moduli at the solitonic level now vary in a controlled way as the internal metric (1.7) evolves in time. Thus at every instant of time the moduli are stabilized with no Dine-Seiberg runaway [50]. We call this dynamical moduli stabilization.

An advantage of choosing M-theory, as against type IIB theory, to do our computations is that we are, in fact, taking into account the full string spectra, which includes the NS and RR sectors of IIB. In M-theory the graviton state, when dimensionally reduced over the 11-dimensional cycle, reproduces the zero mode gravitons. On the other hand, most 
of the massive gravitons coming out of stringy excitations decay at the tree and one-loop level so that they do not contribute to the dynamics of the coherent states. The remaining has been integrated out and they contribute to the infinite tower of perturbative and non-perturbative, including non-local and topological, quantum corrections. Surprisingly, it has been shown that the coherent state is stable under all these contributions from higher order curvature (and flux) terms that come out of the combination of the zero mode gravitons, the instanton gases and the perturbative loops [36]. The action $\mathbf{S}^{(\sigma)}$ above contains all types of quantum corrections, for instance, non-perturbative corrections of the BBS [49] and KKLT [1] type instantons as well as the action of the branes and surfaces, including fermionic interactions, as shown in [36]. Taking this exhaustive array of quantum corrections allowed us to venture to the regime where the curvature could be more than the string scale. In such a scenario, a countable (but large) number of perturbative and nonperturbative quantum corrections become essential [36]. Indeed, this is an important point since the cosmological constant in four-dimensions, in our formalism, emerges from a delicate balancing between the fluxes and the quantum corrections but without any vacuum energies [25]. Furthermore, since the fluctuations are ultimately built out of the interacting vacuum of the solitonic background, perturbative quantum corrections do not lead to any instabilities as everything is expressed in terms of time-independent mode expansions [36], as opposed to the case of classical dS [24, 30]. Although our analyses demonstrates the stability of our state against quantum corrections, we have not shown if the conditions necessary for constructing this Glauber-Sudarshan state are generic or not. Put differently, we have not examined whether this state is an attractor on the space of all such states constructed on top of the warped-Minkowski background, which we leave for future work.

As already mentioned, what makes our solution more physically interesting is that it resolves instabilities related to QFT of fluctuations on top of classical dS spacetime [24], which are not unique to stringy constructions. Remarkably, these fluctuations also turn out to be intimately connected to the entropy of dS spacetime, an old puzzle being the origin of its finiteness within the context of full quantum gravity [9]. Specifically, in our solution, a natural resolution would be in interpreting this as the entanglement entropy between the fluctuation modes, on top of the time-independent solitonic vacuum, which give rise to the Glauber-Sudarshan state itself. The way we get a finite von Neumann entanglement entropy, due to this mode-coupling, is that we have a reduced density matrix corresponding to tracing out the modes which are super-horizon, and treating the causal patch of an inertial observer as our system. The key observation is that if the interactions could be turned off, such an entanglement entropy would consist of diverging parts alone but, in that case, we would have no dS space either as $\mathbf{H}_{\text {int }}$ is crucial in the construction of (2.4). It had been heuristically argued earlier that, for a coherent state description of $\mathrm{dS}$, a finite number of gravitons is synonymous to the finite entropy associated with it; furthermore, it was also noted that interactions between the gravitons are important to arrive at this conclusion [22]. In our case, we sketch out the concrete reason as to how one arrives at a finite number of highly-interacting gravitons (and flux particles) for our Glauber-Sudarshan state necessary for having a finite entropy for the resulting dS space. Our solution also satisfies the expectation of the dS symmetries being emergent for 
a 'reasonably' short time-period, and are not eternal, which is a necessary condition for having a finite entropy $[52,53]$. Without going into details, note that the above procedure is technically tractable due to the fact that the entanglement entropy corresponding to coherent states coincide with that for the vacuum [36, 54].

\section{Conclusion}

To conclude, in this work, we have shown how the EOMs can be solved in full string theory to obtain a dS spacetime by avoiding the swampland, once time-dependent degrees of freedom are turned on, and including quantum corrections, by constructing the solution in terms of a Glauber-Sudarshan state. Remarkably, this state (a) solves the trans-Planckian censorship problem, (b) is stable against both perturbative and non-perturbative quantum corrections, and (c) provides a microscopic understanding of its entropy as an inherently quantum quantity. Furthermore, the order of magnitude of the time-limit before which the system becomes strongly-coupled gives an easy way out of the coincidence problem of cosmology as well as an escape from issues related to Boltzmann brains. This much shorter timescale is a distinguishing feature of our model as opposed to the lifetime of dS in compactifications such as KKLT scenario [1].

\section{Acknowledgments}

We would like to thank Robert Brandenberger for discussions. The work of KD is supported in part by the Natural Sciences and Engineering Research Council of Canada. SB is supported in part by the NSERC (funding reference \#CITA 490888-16) through a CITA National Fellowship and by a McGill Space Institute fellowship.

Open Access. This article is distributed under the terms of the Creative Commons Attribution License (CC-BY 4.0), which permits any use, distribution and reproduction in any medium, provided the original author(s) and source are credited.

\section{References}

[1] S. Kachru, R. Kallosh, A.D. Linde and S.P. Trivedi, de Sitter vacua in string theory, Phys. Rev. D 68 (2003) 046005 [hep-th/0301240] [INSPIRE].

[2] N. Cribiori, R. Kallosh, C. Roupec and T. Wrase, Uplifting Anti-D6-brane, JHEP 12 (2019) 171 [arXiv: 1909.08629] [INSPIRE].

[3] R. Kallosh and A. Linde, Mass Production of Type IIA dS Vacua, JHEP 01 (2020) 169 [arXiv: 1910.08217] [INSPIRE].

[4] A. Linde, KKLT without AdS, JHEP 05 (2020) 076 [arXiv: 2002.01500] [INSPIRE].

[5] G. Obied, H. Ooguri, L. Spodyneiko and C. Vafa, de Sitter Space and the Swampland, arXiv: 1806.08362 [INSPIRE].

[6] P. Agrawal, G. Obied, P.J. Steinhardt and C. Vafa, On the Cosmological Implications of the String Swampland, Phys. Lett. B 784 (2018) 271 [arXiv:1806.09718] [INSPIRE]. 
[7] S.K. Garg and C. Krishnan, Bounds on Slow Roll and the de Sitter Swampland, JHEP 11 (2019) 075 [arXiv: 1807.05193] [INSPIRE].

[8] H. Ooguri, E. Palti, G. Shiu and C. Vafa, Distance and de Sitter Conjectures on the Swampland, Phys. Lett. B 788 (2019) 180 [arXiv:1810.05506] [INSPIRE].

[9] R. Bousso and J. Polchinski, Quantization of four form fluxes and dynamical neutralization of the cosmological constant, JHEP 06 (2000) 006 [hep-th/0004134] [INSPIRE].

[10] S. Weinberg, The Cosmological constant problems, in 4th International Symposium on Sources and Detection of Dark Matter in the Universe (DM 2000), (2000) [astro-ph/0005265] [INSPIRE].

[11] G.W. Gibbons, Thoughts on tachyon cosmology, Class. Quant. Grav. 20 (2003) S321 [hep-th/0301117] [INSPIRE].

[12] G.W. Gibbons, Aspects Of Supergravity Theories, Print-85-0061, Cambridge (1984) [INSPIRE].

[13] J.M. Maldacena and C. Núñez, Supergravity description of field theories on curved manifolds and a no go theorem, Int. J. Mod. Phys. A 16 (2001) 822 [hep-th/0007018] [INSPIRE].

[14] K. Dasgupta, R. Gwyn, E. McDonough, M. Mia and R. Tatar, de Sitter Vacua in Type IIB String Theory: Classical Solutions and Quantum Corrections, JHEP 07 (2014) 054 [arXiv: 1402.5112] [INSPIRE].

[15] U.H. Danielsson and T. Van Riet, What if string theory has no de Sitter vacua?, Int. J. Mod. Phys. D 27 (2018) 1830007 [arXiv:1804.01120] [INSPIRE].

[16] S. Sethi, Supersymmetry Breaking by Fluxes, JHEP 10 (2018) 022 [arXiv:1709.03554] [INSPIRE].

[17] A. Bedroya and C. Vafa, Trans-Planckian Censorship and the Swampland, JHEP 09 (2020) 123 [arXiv:1909.11063] [INSPIRE].

[18] A. Bedroya, R. Brandenberger, M. Loverde and C. Vafa, Trans-Planckian Censorship and Inflationary Cosmology, Phys. Rev. D 101 (2020) 103502 [arXiv:1909.11106] [InSPIRE].

[19] S. Brahma, Trans-Planckian censorship conjecture from the swampland distance conjecture, Phys. Rev. D 101 (2020) 046013 [arXiv:1910.12352] [InSPIRE].

[20] T. Rudelius, Conditions for (No) Eternal Inflation, JCAP 08 (2019) 009 [arXiv: 1905.05198] [INSPIRE].

[21] S. Brahma and S. Shandera, Stochastic eternal inflation is in the swampland, JHEP 11 (2019) 016 [arXiv: 1904.10979] [INSPIRE].

[22] G. Dvali and C. Gomez, On Exclusion of Positive Cosmological Constant, Fortsch. Phys. 67 (2019) 1800092 [arXiv: 1806.10877] [INSPIRE].

[23] J. Martin and R.H. Brandenberger, The TransPlanckian problem of inflationary cosmology, Phys. Rev. D 63 (2001) 123501 [hep-th/0005209] [INSPIRE].

[24] U. Danielsson, The quantum swampland, JHEP 04 (2019) 095 [arXiv: 1809.04512] [INSPIRE].

[25] K. Dasgupta, M. Emelin, E. McDonough and R. Tatar, Quantum Corrections and the de Sitter Swampland Conjecture, JHEP 01 (2019) 145 [arXiv: 1808.07498] [InSPIRE]. 
[26] K. Dasgupta, M. Emelin, M.M. Faruk and R. Tatar, de Sitter Vacua in the String Landscape, arXiv: 1908.05288 [INSPIRE].

[27] K. Dasgupta, M. Emelin, M.M. Faruk and R. Tatar, How a four-dimensional de Sitter solution remains outside the swampland, arXiv:1911.02604 [INSPIRE].

[28] K. Dasgupta, M. Emelin, M. Mehedi Faruk and R. Tatar, de Sitter Vacua in the String landscape: La Petite Version, in 11th International Symposium on Quantum Theory and Symmetries, (2019) [arXiv:1911.12382] [INSPIRE].

[29] M. Emelin, Effective Theories as Truncated Trans-Series and Scale Separated Compactifications, JHEP 11 (2020) 144 [arXiv: 2005.11421] [INSPIRE].

[30] E. Palti, The Swampland: Introduction and Review, Fortsch. Phys. 67 (2019) 1900037 [arXiv: 1903.06239] [INSPIRE].

[31] G. Dvali and C. Gomez, Black Hole's Quantum N-Portrait, Fortsch. Phys. 61 (2013) 742 [arXiv:1112.3359] [INSPIRE].

[32] G. Dvali and C. Gomez, Quantum Compositeness of Gravity: Black Holes, AdS and Inflation, JCAP 01 (2014) 023 [arXiv:1312.4795] [INSPIRE].

[33] G. Dvali, C. Gomez, R.S. Isermann, D. Lüst and S. Stieberger, Black hole formation and classicalization in ultra-Planckian $2 \rightarrow N$ scattering, Nucl. Phys. B 893 (2015) 187 [arXiv: 1409.7405] [INSPIRE].

[34] G. Dvali, C. Gomez and S. Zell, Quantum Break-Time of de Sitter, JCAP 06 (2017) 028 [arXiv: 1701.08776] [INSPIRE].

[35] G. Dvali, C. Gomez and S. Zell, Quantum Breaking Bound on de Sitter and Swampland, Fortsch. Phys. 67 (2019) 1800094 [arXiv:1810.11002] [INSPIRE].

[36] S. Brahma, K. Dasgupta and R. Tatar, de Sitter Space as a Glauber-Sudarshan State, JHEP 02 (2021) 104 [arXiv:2007.11611] [INSPIRE].

[37] H.E.S. Velten, R.F. vom Marttens and W. Zimdahl, Aspects of the cosmological "coincidence problem", Eur. Phys. J. C 74 (2014) 3160 [arXiv:1410.2509] [INSPIRE].

[38] E. Witten, On flux quantization in M-theory and the effective action, J. Geom. Phys. 22 (1997) 1 [hep-th/9609122] [INSPIRE].

[39] E.C.G. Sudarshan, Equivalence of semiclassical and quantum mechanical descriptions of statistical light beams, Phys. Rev. Lett. 10 (1963) 277 [INSPIRE].

[40] R.J. Glauber, Coherent and incoherent states of the radiation field, Phys. Rev. 131 (1963) 2766 [INSPIRE].

[41] A. Sen, F theory and orientifolds, Nucl. Phys. B 475 (1996) 562 [hep-th/9605150] [InSPIRE].

[42] K. Dasgupta and S. Mukhi, F theory at constant coupling, Phys. Lett. B 385 (1996) 125 [hep-th/9606044] [INSPIRE].

[43] K. Becker and M. Becker, M theory on eight manifolds, Nucl. Phys. B 477 (1996) 155 [hep-th/9605053] [INSPIRE].

[44] K. Dasgupta, G. Rajesh and S. Sethi, M theory, orientifolds and G - flux, JHEP 08 (1999) 023 [hep-th/9908088] [inSPIRE].

[45] G.S. Agarwal and K. Tara, Nonclassical properties of states generated by the excitations on a coherent state, Phys. Rev. A 43 (1991) 492 [InSPIRE]. 
[46] F.J. Dyson, The S matrix in quantum electrodynamics, Phys. Rev. 75 (1949) 1736 [INSPIRE].

[47] J.S. Schwinger, On the Green's functions of quantized fields. 1, Proc. Nat. Acad. Sci. 37 (1951) 452 [INSPIRE].

[48] J.S. Schwinger, On the Green's functions of quantized fields. 2, Proc. Nat. Acad. Sci. 37 (1951) 455 [INSPIRE].

[49] K. Becker, M. Becker and A. Strominger, Five-branes, membranes and nonperturbative string theory, Nucl. Phys. B 456 (1995) 130 [hep-th/9507158] [INSPIRE].

[50] M. Dine and N. Seiberg, Is the Superstring Weakly Coupled?, Phys. Lett. B 162 (1985) 299 [INSPIRE].

[51] R. Bousso, Adventures in de Sitter space, in Workshop on Conference on the Future of Theoretical Physics and Cosmology in Honor of Steven Hawking's 60th Birthday, (2002) [hep-th/0205177] [INSPIRE].

[52] N. Goheer, M. Kleban and L. Susskind, The Trouble with de Sitter space, JHEP 07 (2003) 056 [hep-th/0212209] [INSPIRE].

[53] N. Arkani-Hamed, S. Dubovsky, A. Nicolis, E. Trincherini and G. Villadoro, A Measure of de Sitter entropy and eternal inflation, JHEP 05 (2007) 055 [arXiv: 0704.1814] [INSPIRE].

[54] E. Benedict and S.-Y. Pi, Entanglement entropy of nontrivial states, Annals Phys. 245 (1996) 209 [hep-th/9505121] [INSPIRE]. 